\title{
AVALIAÇÃO DA HABILIDADE DE RESOLUÇÃO TEMPORAL, COM USO DO TOM PURO, EM CRIANÇAS COM E SEM DESVIO FONOLÓGICO
}

\section{Temporal processes ability evaluations with pure tones in children with and with no phonological disorders}

\author{
Lílian Ferreira Muniz ${ }^{(1)}$, Antônio Roazzi (2), Eliane Schochat ${ }^{(3)}$, \\ Cleide Fernandes Teixeira ${ }^{(4)}$, Jônia Alves de Lucena ${ }^{(5)}$
}

\begin{abstract}
RESUMO
Objetivo: verificar o desempenho da resolução temporal em crianças portadoras de desvio fonológico. Métodos: a área de estudo foi uma clínica escola de Fonoaudiologia de uma universidade particular do Recife e teve como participantes 36 crianças, de 6 a 9 anos, subdivididas em um grupo experimental (com desvio fonológico) e um grupo controle. Para a coleta de dados foi utilizado o Teste de detecção de intervalos aleatórios proposto por Keith e a análise quantitativa dos dados foi realizada com o SPSS 13 para a aplicação de técnicas de estatística descritiva. Resultados: 94,5\% das crianças com desvio fonológico apresentaram resultados alterados para o RGDT, os resultados mostram limiares de detecção de intervalos aleatórios maiores no grupo com desvio fonológico $(25,00$ a $28,33 \mathrm{~ms}$ ); tendo sido significativa a diferença entre grupos para cada uma das freqüências testadas; não houve influência das variáveis: freqüência, sexo, idade e série escolar; o tratamento fonoaudiológico não foi um fator determinante; as queixas de aprendizagem mais referidas foram: dificuldade de leitura e dificuldade de compreensão de textos. Conclusão: crianças com desvio fonológico podem apresentar alteração de processamento temporal e necessitam de mais tempo para detecção de intervalos de tempo entre estímulos auditivos que as crianças sem desvio fonológico. De maneira geral, em ambos os grupos, não houve influência das variáveis: freqüência, sexo, idade, série escolar ou ocorrência de tratamento fonoaudiológico. As queixas de aprendizado podem estar presentes nas crianças com desvio fonológico e alteração de processamento temporal.
\end{abstract}

DESCRITORES: Audição; Perda Auditiva; Percepção Auditiva

\section{INTRODUÇÃO}

A audição é uma das funções sensoriais que facilita o contato do indivíduo com o meio ambiente e tem papel fundamental na sua integração à sociedade ${ }^{1}$.

(1) Fonoaudióloga; Professora Assistente da Universidade Católica de Pernambuco; Doutora em Psicologia Cognitiva.

(2) Psicólogo; Professor Associado I da Universidade Federal de Pernambuco; Doutor em Psicologia.

(3) Fonoaudióloga; Professora Assistente Doutor da Universidade de São Paulo; Doutora em Lingüística.

(4) Fonoaudióloga; Professora Assistente da Faculdade Integrada do Recife; Doutora em Saúde Pública.

(5) Fonoaudióloga; Professora Assistente da Universidade Católica de Pernambuco; Doutora em Psicologia Cognitiva.
Os componentes neurobiológicos da audição envolvem uma complexidade de eventos e uma enormidade de inter-relações no sistema nervoso central ${ }^{2}$. Os processos e mecanismos do sistema auditivo relacionam-se com a percepção dos sinais verbais e não verbais da linguagem influenciando as funções mais elevadas do aprendizado ${ }^{3}$. Ao longo das vias auditivas, envolvem a participação de redes neuronais complexas, assim como das funções mentais superiores. Desvios ou dificuldades em determinadas conexões podem frustrar ou mesmo impedir o aprendizado de certos conhecimentos que têm como estímulo o som.

O processamento auditivo central é conceituado como sendo os mecanismos e processos do sistema auditivo responsáveis pelos seguintes 
fenômenos comportamentais: localização e lateralização sonora, discriminação auditiva, reconhecimento de padrões auditivos, aspectos temporais da audição (resolução temporal, mascaramento temporal, integração temporal e ordenação temporal), desempenho auditivo na presença de sinais acústicos competitivos e desempenho auditivo para sinais acústicos degradados ${ }^{4}$.

O processo de aprendizado sonoro envolve, entre outras coisas, o conhecimento das propriedades do som: altura, intensidade, duração e timbre, além do conceito de pulsação, melodia, ritmo, harmonia e forma. A percepção auditiva, parte do processamento auditivo, precisa ser estimulada, pois o desenvolvimento auditivo depende de estímulos constantes e progressivos, favorecendo a compreensão de qualquer som verbal, bem como dos sons não-verbais e das informações supra-segmentais presentes na fala. Para compreensão do que é dito faz-se necessário o perfeito funcionamento do sistema auditivo central ${ }^{5-7}$.

São muitos os detalhes envolvidos neste tipo de análise e nem sempre é possível identificar os órgãos responsáveis para cada tarefa na percepção auditiva, assim sendo, a neurociência auditiva vem trabalhando com o objetivo de identificar as várias habilidades auditivas e seus substratos neurológicos. Dentre as habilidades auditivas já identificadas encontra-se a temporalidade ${ }^{8}$.

A habilidade para produzir fala inteligível depende, em grande parte, das habilidades para processar os paradigmas de espectro acústico e da prosódia da fala do locutor ${ }^{9}$. Existe uma associação direta entre a percepção acústica temporal e percepção da fala. Dificuldades em perceber mudanças rápidas no sinal acústico influenciam tanto a percepção do fonema como os aspectos mais abrangentes relacionados ao reconhecimento da fala ${ }^{6}$. A seqüencialização temporal envolve percepção e/ou processamento de dois ou mais estímulos auditivos em sua ordem de ocorrência no tempo. É uma das mais básicas e importantes funções do sistema nervoso auditivo central ${ }^{10}$ e que envolve áreas inter e intra-hemisféricas ${ }^{11}$.

As implicações das alterações do processamento auditivo de ordem temporal ou não, são inúmeras; mesmo o atraso nas etapas de maturação do processamento auditivo pode ser um fator preditivo de desvio no desenvolvimento da linguagem ${ }^{12}$.

O transtorno do processamento auditivo se refere a um distúrbio da audição em que há impedimento da habilidade de analisar e/ou interpretar padrões sonoros. Tal como qualquer outra atividade mental, resulta da falha de uma ou mais unidades, na realização de tarefas ou operações, podendo se manifestar de forma muito variada, uma vez que é de natureza multifatorial ${ }^{13,14}$. Seria então uma deficiência em uma ou mais habilidades auditivas que para alguns é o resultado de uma disfunção destes processos e mecanismos dedicados à audição; para outros pode resultar de outras disfunções mais gerais, como déficit de atenção, déficit de timing nervoso e é possível que reflita disfunções coexistentes de ambos os tipos ${ }^{8}$.

A avaliação do processamento auditivo é multidimensional, devendo incorporar tanto a análise acústica do sinal quanto os processos cognitivos superiores. Este é um procedimento que tem como objetivo determinar a presença ou não de um transtorno, descrever os parâmetros de extensão, avaliar os transtornos de maturação do sistema nervoso central, detectar e identificar o local da disfunção auditiva central, ressaltar as habilidades preferenciais de aprendizagem, estabelecer diretrizes e critérios para a elaboração do programa de reabilitação, avaliar benefícios de tratamentos (médicos, cirúrgicos e educacionais) e determinar o encaminhamento a outros profissionais. Pode ser realizada por meio de testes eletrofisiológicos e comportamentais, os quais se complementam para descrever a função das áreas cerebrais envolvidas neste processo ${ }^{15}$. Porém, os testes comportamentais trazem maior riqueza de informações acerca da natureza da ação das respostas do indivíduo ${ }^{16}$.

Estes testes comportamentais podem ser agrupados segundo a forma de apresentação do estímulo. Existem testes dióticos (estímulos diferentes enviados simultaneamente, um a cada orelha), monóticos (estímulo(s) enviado apenas a uma orelha) e dicóticos (estímulos diferentes chegam às duas orelhas ao mesmo tempo) para avaliação do processamento auditivo, porém os testes dióticos não avaliam as orelhas separadamente; não utilizam fones; os estímulos utilizados podem ser a própria voz do avaliador ou objetos sonoros. A apresentação binaural equivaleria à apresentação diótica e a monoaural apresentação monótica 9 . Dentre os testes dicóticos encontra-se o RGDT (Random Gap Detection Test), um teste de detecção de intervalos (Gaps) aleatório. Qualquer sujeito com limiar de detecção dos Gaps aleatórios maior que $20 \mathrm{msec}$, provavelmente, apresenta transtorno de processamento temporal que interfere na percepção da fala normal e reconhecimento de fonemas. Quanto maior for o Gap, em milisegundo, maior a probabilidade do transtorno do processamento temporal interferir na percepção da fala, sendo o limiar de detecção de Gap definido como o intervalo de tempo no qual o sujeito, conscientemente, identifica dois tons ${ }^{17,18}$.

As alterações do processamento auditivo podem estar contidas em quadros de distúrbios de lingua- 
gem, piorando o desempenho de indivíduos em tarefas de compreensão do som que requeiram habilidades auditivas, assim como, tais alterações podem atuar como causa das dificuldades de linguagem que ocorrem quando o indivíduo falha ao receber ou resgatar o que foi ouvido ${ }^{19}$. Estas alterações podem ocasionar habilidades limitadas em identificar alguns elementos fonéticos breves, apresentados em contextos de fala e um desempenho baixo em identificar ou seqüencializar os estímulos acústicos de curta duração apresentados em seqüência rápida ${ }^{20}$.

As dificuldades de aprendizado da linguagem oral parecem ser atribuídas a uma inabilidade em processar rapidamente mudanças nas pistas acústicas da fala fluente, bem como a dificuldades na discriminação fonológica que é uma função do sistema do processamento central e que se desenvolve muito cedo. Em conseqüência desta dificuldade pode surgir, mais tarde, dificuldade de leitura, escrita e na habilidade de soletrar ${ }^{21}$. A percepção auditiva tem sido relatada como uma das fontes de variação individual das habilidades fonológicas, que tem papel fundamental no aprendizado ${ }^{22}$.

Parece certo que prejuízos sutis no processamento auditivo das informações podem levar ao desenvolvimento de representações alteradas de informação verbal na memória e, ainda, a dificuldades de segmentação fonêmica de palavras, sendo que alteração da percepção auditiva não verbal pode afetar as habilidades fonológicas ${ }^{23,24}$. Os padrões temporais e espectrais da fala são armazenados na memória sensorial por um breve período de tempo no qual é realizada a análise. $\mathrm{Na}$ habilidade para ouvir a fala, o ouvinte pode contar com redundâncias intrínsecas (múltiplas vias e tratos auditivos do sistema nervoso auditivo central) e extrínsecas (numerosas pistas sobrepostas dentro da própria fala). O ouvinte não necessitaria de todas as pistas para compreender a mensagem a todo o momento, pois o sinal da fala é muito redundante. Mas, para crianças que estão em fase de desenvolvimento da estrutura da língua seria necessária uma adequada recepção dos aspectos acústicos da fala ${ }^{25}$.

A prosódia e a entonação trazem emoção ao que está sendo falado (alegria, raiva, entusiasmo, surpresa, etc.) e podem mudar o significado da sentença. Uma vez que os indivíduos que apresentam processamento auditivo alterado não possuem uma boa discriminação de freqüência, intensidade e duração sonora, a prosódia e a entonação do que é falado provavelmente não são apreciadas por completo ${ }^{26}$.

$O$ interesse em estudar as habilidades auditivas de crianças, correlacionado-as às habilidades de linguagem e aprendizado, vem aumentando nos últimos anos. Muitos problemas de linguagem, fala e aprendizado vêm sendo atribuídos às dificuldades no processamento do sinal acústico ${ }^{27}$.

O desvio fonológico indica dificuldades potenciais em diversos níveis, tais como: a discriminação de diferentes fonemas; o reconhecimento de contrastes fonológicos e das representações desses contrastes no léxico; a modificação dos sons que são padrões na fala, em razão do uso inadequado das regras fonológicas; e a articulação imprecisa, entre outros fatores ${ }^{28}$. A causa do desvio fonológico é desconhecida e a severidade da inteligibilidade da fala apresenta graus variados ${ }^{28}$, afetando $10 \%$ da população em idade pré-escolar nos Estados Unidos, assim como no Brasil ${ }^{29,30}$.

Independentemente da língua a que a criança está exposta, à medida que ela adquire regras fonológicas mais importantes, mantendo os contrastes distintivos, aumenta a inteligibilidade da fala e, com isso, as suas possibilidades de comunicação e convívio social ${ }^{28,31,32}$.

A presença de alterações do sistema auditivo de ordem central em portadores de distúrbios de aquisição de linguagem aponta para a relação destes com as funções cognitivas, lingüísticas e sociais associadas ao aprendizado e ao uso da linguagem ${ }^{5-7}$.

Assim, além dos estudos das teorias que envolvem as regras lingüísticas, explicativas da natureza da desordem da fala e o seu tratamento, tornam-se importantes os estudos que abordem a fisiologia da interpretação dos eventos acústicos. Tais estudos devem fundamentar, cada vez mais, os trabalhos de pesquisas relacionados à percepção da fala. Os testes que representam a avaliação comportamental da percepção auditiva ultrapassam a sensação e envolvem sempre, em algum grau, a atenção, memória ou cognição, sendo de fundamental importância para a melhor compreensão das alterações de linguagem.

Existem poucos centros no Brasil que se dedicam a estes estudos e estes estão em fase inicial em suas pesquisas. Mesmo a literatura internacional é recente e rara neste assunto ${ }^{33}$.

Os objetivos deste estudo foram verificar o desempenho do processamento temporal de crianças portadoras de desvio fonológico; comparar este desempenho ao de um grupo controle; caracterizar os resultados segundo freqüência, sexo, idade, série escolar e ocorrência de tratamento fonoaudiológico e verificar, no grupo portador de desvio fonológico e alteração de processamento temporal, a ocorrência de dificuldades de aprendizagem. 


\section{MÉTODOS}

Trata-se de estudo descritivo, observacional e de corte transversal e foi realizado em uma clínicaescola de Fonoaudiologia de uma universidade privada, localizada na cidade do Recife. Os participantes deste estudo foram crianças, voluntárias, de ambos os sexos, as quais foram divididas em dois grupos. Os critérios de inclusão para o grupo 1 foram: ter diagnóstico fonoaudiológico de desvio fonológico; ser aluno da rede pública de ensino; ter idade de 6 a 9 anos; apresentar o português como primeira língua; ter acuidade auditiva normal, limiares tonais por via aérea de até $20 \mathrm{~dB}$ NA para as freqüências de 500 a $4000 \mathrm{~Hz}{ }^{4}$; não ser portador distúrbios ou síndromes que comprometam a compreensão dos testes, bem como o desenvolvimento lingüístico; apresentar timpanogramas do tipo A ${ }^{34}$; ter reflexos estapedianos presentes, na pesquisa ispi e contralateral, em todas as freqüências e ter limiares de detecção de Gap menor que 20 milissegundos (ms) para o sub-teste 1 do RGDT proposto por Keith ${ }^{17}$.

Como critérios de inclusão para o grupo 2 foram usados os mesmos acima mencionados, com exceção do diagnóstico do desvio fonológico e ainda, como critério de exclusão, apresentar qualquer alteração de linguagem oral.

Foram excluídos, em ambos os grupos, os participantes com qualquer tipo de treinamento musical, pois este pode interferir no desenvolvimento do processamento auditivo temporal e, ainda, os indivíduos que fizessem uso de medicação com atuação sobre o sistema nervoso central. Os participantes foram pareados por: idade, sexo, série escolar, nível social, nível econômico e cultural. Para a seleção dos participantes foi realizado um levantamento no banco de dados da clínica-escola e para cada criança com desvio fonológico foi selecionada, em sua mesma escola, uma criança com as características mencionadas para o grupo 2. Ambos os grupos foram constituídos por 18 crianças, perfazendo um número total de 36 participantes. A distribuição foi a mesma por sexo, faixa etária e série escolar, em cada grupo, sendo: 10 do sexo feminino e oito do sexo masculino, 7 na faixa etária de 6 a 7 anos e 11 na faixa de 8 a 9 anos e 5 na alfabetização, 7 na primeira série, 4 na segunda série e 2 na terceira série.

Após o levantamento dos participantes na clínica escola, foi realizado contato com as escolas da rede pública de ensino, quando foi encaminhada uma carta de apresentação. Apresentou-se uma carta de esclarecimento aos pais ou responsáveis para a obtenção do consentimento e com a assinatura do termo de consentimento livre e esclarecido foi aplicada uma anamnese, baseada nos modelos de Pereira ${ }^{13}$, realizou-se uma meatoscopia com otoscópio WELCH ALLYN 29000, a imitanciometria que constou da avaliação timpanométrica e da pesquisa de reflexos estapedianos ipsilaterais em 1000 e $2000 \mathrm{~Hz}$, bem como contralaterais em 500, 1000, 2000 e $4000 \mathrm{~Hz}^{34}$, a audiometria tonal realizada nas freqüências de 500 a $4000 \mathrm{~Hz}$, com técnica descendente, em escala de $10 \mathrm{~dB} \mathrm{NA}{ }^{4}$ com o audiômetro da marca $A M P L A I D$, modelo 460 , com o qual foi avaliado também o processamento auditivo temporal e a audiometria vocal (índice percentual de reconhecimento de fala e limiar de reconhecimento de fala). Os fones utilizados nos procedimentos audiológicos foram os supraurais, TDH 39. Acoplado ao audiômetro, havia ainda um toca CD (compact disc) da marca Panasonic, modelo $S 35$ que permitiu a aplicação do teste específico para o processamento auditivo temporal.

A imitanciometria, realizada como o imitanciômetro AZ7 da marca INTERACOUSTICS, constou da avaliação timpanométrica e pesquisa de reflexos estapedianos ipsilaterais em 1000 e $2000 \mathrm{~Hz}$, bem como contralaterais em 500, 1000, 2000 e $4000 \mathrm{~Hz}^{34}$.

O teste de processamento temporal (avaliação auditiva central) foi o RGDT, proposto por Keith ${ }^{17}$, realizado com material gravado em $\mathrm{CD}$, em um nível de intensidade referida como confortável pelo participante.

Inicialmente foi realizado o sub-teste 1 , com a finalidade de garantir a compreensão do procedimento (seleção dos sujeitos) e o sub-teste 2 para avaliação do processamento temporal (coleta de dados). Foram representados pares de cliques com duração de 17 milissegundos (ms), com objetivo de caracterizar o intervalo de tempo (Gaps) em milissegundos para o qual o indivíduo identificou a presença de dois tons. Nos dois sub-testes houve uma variação aleatória de tempo dos Gaps de 0 a 40 $\mathrm{ms}$, porém o sub-teste 1 foi realizado apenas em $500 \mathrm{~Hz}$, não havendo mudança no padrão de freqüência; no entanto, o sub-teste 2 foi realizado nas freqüências de $500,1000,2000$ e $4000 \mathrm{~Hz}$. O teste central durou cerca de 10 minutos, sendo ambos os sub-testes registrados em formulários específicos (Anexo A). Limiares de detecção de Gap com respostas de até 20 milissegundos (ms), por freqüência, são considerados normais. Para que não haja evidência da existência de transtorno no processamento temporal é necessário apresentar 3 das quatro frequências analisadas dentro dos padrões normais. Foi calculado ainda o tempo de detecção composto para os Gaps aleatórios, realizando-se a média de respostas obtidas nas quatro frequências testadas ${ }^{17}$. Tanto a audiometria, quanto o RGDT 
foram realizados em cabina acústica e os materiais usados para as avaliações audiológicas (periférica e central) seguiram resoluções de calibração propostas pela ASHA ${ }^{4}$. Para a análise de dados foi utilizado o programa estatístico SPSS (Statistical Package for Social Sciences) 13 e o nível de significância utilizado nas decisões dos testes estatísticos foi de $5,0 \%$. A apresentação dos dados foi realizada de forma tabular e gráfica.

O projeto foi encaminhado ao Comitê de Ética em Pesquisa da Universidade Católica de Pernambuco tendo sido aprovado com CEP 049/2005.

\section{RESULTADOS}

Analisando o teste de detecção de Gaps aleatórios, no grupo de crianças portadoras de desvio fonológico, observa-se que a grande maioria das crianças $(94,5 \%)$ apresentou resultados alterados para o RGDT, quando comparada ao padrão normativo estabelecido para este teste ${ }^{17}$ que é de até 20 ms. O oposto aconteceu com o grupo 2, que apresentou a maior parte das crianças $(83,33 \%)$ com tempo de detecção de Gaps inferior a $20 \mathrm{~ms}$.

Destaca-se na Tabela 1 que a média dos limiares de detecção de Gaps aleatórios variou de 9,78 a 10,72 ms no grupo normal (grupo 2) e de 25,00 a 28,33 ms no grupo com desvio fonológico (grupo 1), para cada uma das freqüências. Analisando-se a média de respostas, encontrou-se maior tempo de detecção de Gaps na freqüência de $4000 \mathrm{~Hz}$ para o grupo 2 e a menor média obtida foi igual para as freqüências de 1000 e $2000 \mathrm{~Hz}$. Para o grupo1, a maior média foi obtida em $1000 \mathrm{~Hz}$ e a menor em $500 \mathrm{~Hz}$.

Tabela 1 - Estatísticas do tempo de detecção de Gaps aleatórios, segundo o grupo, por freqüência testada

\begin{tabular}{|c|c|c|c|c|}
\hline \multirow[b]{2}{*}{ Freqüência } & \multirow[b]{2}{*}{ Estatísticas } & \multicolumn{2}{|c|}{ Grupo } & \multirow[b]{2}{*}{ Valor de $p$} \\
\hline & & $\begin{array}{l}\text { Normal } \\
(n=18)\end{array}$ & $\begin{array}{c}\text { Com desvio } \\
(\mathrm{n}=18)\end{array}$ & \\
\hline \multirow[t]{5}{*}{ - $500 \mathrm{~Hz}$} & Média & 10,39 & 25,00 & $\mathrm{p}^{(1)}<0,001^{*}$ \\
\hline & Desvio padrão & 5,07 & 4,54 & \\
\hline & Coeficiente de variação & 48,80 & 18,16 & \\
\hline & Mínimo & 2,00 & 15,00 & \\
\hline & Máximo & 20,00 & 30,00 & \\
\hline \multirow[t]{5}{*}{ - $1000 \mathrm{~Hz}$} & Média & 9,78 & 28,33 & $\mathrm{p}^{(1)}<0,001^{*}$ \\
\hline & Desvio padrão & 6,14 & 8,91 & \\
\hline & Coeficiente de variação & 62,78 & 31,45 & \\
\hline & Mínimo & 2,00 & 15,00 & \\
\hline & Máximo & 20,00 & 40,00 & \\
\hline \multirow{5}{*}{ - $2000 \mathrm{~Hz}$} & Média & 9,78 & 26,94 & $\mathrm{p}^{(1)}<0,001^{*}$ \\
\hline & Desvio padrão & 5,90 & 6,89 & \\
\hline & Coeficiente de variação & 60,33 & 25,58 & \\
\hline & Mínimo & 2,00 & 20,00 & \\
\hline & Máximo & 20,00 & 40,00 & \\
\hline \multirow[t]{5}{*}{ - $4000 \mathrm{~Hz}$} & Média & 10,72 & 25,83 & $\mathrm{p}^{(1)}<0,001^{*}$ \\
\hline & Desvio padrão & 9,77 & 8,62 & \\
\hline & Coeficiente de variação & 91,14 & 33,37 & \\
\hline & Mínimo & 2,00 & 5,00 & \\
\hline & Máximo & 40,00 & 40,00 & \\
\hline \multirow[t]{5}{*}{ - Gap composto } & Média & 10,16 & 26,15 & $\mathrm{p}^{(1)}<0,001^{*}$ \\
\hline & Desvio padrão & 3,85 & 4,97 & \\
\hline & Coeficiente de variação & 37,89 & 19,00 & \\
\hline & Mínimo & 4,75 & 15,00 & \\
\hline & Máximo & 17,50 & 33,75 & \\
\hline
\end{tabular}

$\left(^{*}\right)$ - Diferença significante ao nível de 5,0\%.

(1) - Através do teste Mann-Whitney 
Tabela 2 - Média e desvio padrão do tempo de detecção de Gaps, segundo o grupo e o sexo por freqüência

\begin{tabular}{|c|c|c|c|}
\hline \multirow{3}{*}{ Freqüência } & \multirow{3}{*}{ Sexo } & \multicolumn{2}{|c|}{ Grupo } \\
\hline & & Normal & Com desvio \\
\hline & & Média \pm D.P & Média \pm D.P \\
\hline \multirow[t]{2}{*}{ - $500 \mathrm{~Hz}$} & Feminino & $10,00 \pm 4,71^{(1)}$ & $25,50 \pm 2,84$ \\
\hline & Masculino & $10,88 \pm 5,77$ & $24,38 \pm 6,23$ \\
\hline \multicolumn{2}{|l|}{ Valor de $p$} & $\mathrm{p}^{(2)}=0,727$ & $\mathrm{p}^{(2)}=0,616$ \\
\hline \multirow[t]{2}{*}{ • $1000 \mathrm{~Hz}$} & Feminino & $10,70 \pm 6,53$ & $31,00 \pm 9,66$ \\
\hline & Masculino & $8,63 \pm 5,83$ & $25,00 \pm 7,07$ \\
\hline \multicolumn{2}{|l|}{ Valor de $p$} & $\mathrm{p}^{(2)}=0,493$ & $\mathrm{p}^{(2)}=0,148$ \\
\hline \multirow{2}{*}{ • $2000 \mathrm{~Hz}$} & Feminino & $8,90 \pm 5,69$ & $27,50 \pm 5,40$ \\
\hline & Masculino & $10,88 \pm 6,36$ & $26,25 \pm 8,76$ \\
\hline \multicolumn{2}{|l|}{ Valor de $p$} & $\mathrm{p}^{(2)}=0,497$ & $\mathrm{p}^{(2)}=0,714$ \\
\hline \multirow[t]{2}{*}{ - $4000 \mathrm{~Hz}$} & Feminino & $8,90 \pm 7,00$ & $27,50 \pm 7,17$ \\
\hline & Masculino & $13,00 \pm 12,57$ & $23,75 \pm 10,26$ \\
\hline Valor de $p$ & & $\mathrm{p}^{(2)}=0,392$ & $\mathrm{p}^{(2)}=0,375$ \\
\hline
\end{tabular}

(1) - Resultados obtidos de 10 pesquisados do sexo masculino e 8 do sexo feminino em cada grupo

(2) - Através do teste Mann-Witney

$\mathrm{Na}$ análise entre os grupos observa-se uma variação do comportamento do limiar de detecção de Gaps, no que se refere ao tempo e à freqüência, sendo possível destacar que houve diferença significativa entre as médias dos grupos, para cada uma das freqüências testadas $(p<0,05)$.

Foram obtidos ainda os Gaps compostos, que representam uma média geral das respostas obtidas para todas as freqüências avaliadas. Na Tabela 1, observa-se média de Gap composto de 10,16ms para o grupo 2 e de $26,15 \mathrm{~ms}$ para o grupo $1 \mathrm{e}$ comprova-se diferença significativa entre os grupos $(p<0,05)$.

Na Tabela 2 apresentam-se a média e o desvio padrão da detecção de Gaps segundo o grupo e o sexo, para cada uma das freqüências analisadas. Desta tabela destaca-se que no grupo normal, com exceção da freqüência de $1000 \mathrm{~Hz}$, a média do processamento temporal foi mais elevada entre os pesquisados do sexo masculino do que os do sexo feminino; no grupo com desvio fonológico as médias foram correspondentemente menos elevadas entre os pesquisados no sexo feminino do que entre os do sexo masculino para cada uma das freqüências, entretanto, para nenhum dos grupos e freqüências comprova-se diferença significativa entre os sexos para o nível de significância fixado $(p>0,05)$.

$\mathrm{Na}$ Tabela 3 apresentam-se a média e o desvio padrão do processamento temporal (tempo de detecção de Gaps aleatório), segundo o grupo e a faixa etária, para cada uma das freqüências ana- lisadas. Desta tabela destaca-se com exceção da freqüência de $2000 \mathrm{~Hz}$, no grupo com desvio, para as demais comparações, em ambos os grupos, não se comprova diferença significativa.

$\mathrm{Na}$ Tabela 4, apresentam-se a média e desvio padrão do tempo de detecção de Gaps segundo a série escolar, por grupo. Desta tabela destaca-se que, no grupo normal, a média do tempo analisado foi mais elevada entre as crianças da alfabetização do que as crianças das outras séries. No grupo com desvio, não se caracteriza nenhum comportamento destacável das médias entre as séries para nenhuma das freqüências.

$\mathrm{Na}$ Tabela 5, analisa-se a média e o desvio padrão segundo a condição: ter feito tratamento fonoaudiológico ou não, no grupo com desvio fonológico. Nesta tabela verifica-se que com exceção da freqüência de $500 \mathrm{~Hz}$ que apresentou médias exatamente iguais em ambos os grupos, para as demais freqüências, as médias do tempo foram correspondentemente mais elevadas no grupo dos que não fizeram tratamento fonoaudiológico; porém, para nenhuma das freqüências comprovam-se diferenças significativas do ponto de vista estatístico.

Na Figura 1, observa-se que dentre os portadores de desvio fonológico que apresentam alteração de processamento temporal, 17 crianças das 18 pertencentes ao grupo experimental, 14 crianças referiram dificuldades de aprendizado. Algumas delas referiram mais de uma das dificuldades pesquisadas e apenas 3 não apresentaram qualquer 
Tabela 3 - Média e desvio padrão do processamento temporal segundo o grupo e a faixa etária por freqüência

\begin{tabular}{|c|c|c|c|}
\hline \multirow{3}{*}{ Freqüência } & \multirow{3}{*}{ Faixa etária (em anos) } & \multicolumn{2}{|c|}{ Grupo } \\
\hline & & Normal & Com desvio \\
\hline & & Média \pm D.P & Média \pm D.P \\
\hline \multirow[t]{2}{*}{ - $500 \mathrm{~Hz}$} & 6 a 7 & $10,29 \pm 5,22^{(1)}$ & $25,00 \pm 5,00$ \\
\hline & 8 a 9 & $10,45 \pm 5,22$ & $25,00 \pm 4,47$ \\
\hline \multicolumn{2}{|l|}{ Valor de $p$} & $p^{(2)}=0,948$ & $p^{(2)}=1,000$ \\
\hline \multirow[t]{2}{*}{ • $1000 \mathrm{~Hz}$} & 6 a 7 & $10,29 \pm 6,63$ & $27,86 \pm 9,06$ \\
\hline & 8 a 9 & $9,45 \pm 6,12$ & $28,64 \pm 9,24$ \\
\hline \multicolumn{2}{|l|}{ Valor de $p$} & $p^{(2)}=0,789$ & $p^{(2)}=0,863$ \\
\hline \multirow[t]{2}{*}{ • $2000 \mathrm{~Hz}$} & 6 a 7 & $11,43 \pm 4,76$ & $22,86 \pm 3,93$ \\
\hline & 8 a 9 & $8,73 \pm 6,51$ & $29,55 \pm 7,23$ \\
\hline \multicolumn{2}{|l|}{ Valor de $p$} & $p^{(2)}=0,359$ & $p^{(2)}=0,040^{*}$ \\
\hline \multirow[t]{2}{*}{ - $4000 \mathrm{~Hz}$} & 6 a 7 & $13,86 \pm 13,13$ & $22,86 \pm 10,75$ \\
\hline & 8 a 9 & $8,73 \pm 6,89$ & $27,73 \pm 6,84$ \\
\hline Valor de $p$ & & $p^{(2)}=0,291$ & $p^{(2)}=0,254$ \\
\hline
\end{tabular}

$\left(^{*}\right)$ - Diferença significante a 5,0\%.

(1) - Resultados obtidos de 7 pesquisados na faixa de 6 a 7 anos e 11 na faixa de 8 a 9 em cada grupo

(2) - Através do teste Kruskal-Wallis.

Tabela 4 - Média e desvio padrão do tempo de processamento segundo o grupo e a série escolar

\begin{tabular}{|c|c|c|c|}
\hline \multirow{3}{*}{ Freqüência } & \multirow{3}{*}{ Série que freqüentava } & \multicolumn{2}{|c|}{ Grupo } \\
\hline & & Normal & Com desvio \\
\hline & & Média \pm D.P & Média \pm D.P \\
\hline \multirow[t]{3}{*}{ • $500 \mathrm{~Hz}$} & Alfabetização & $11,00 \pm 5,48$ & $24,00 \pm 5,48$ \\
\hline & Primeira & $9,57 \pm 4,79$ & $25,00 \pm 5,00$ \\
\hline & Segunda/terceira & $10,83 \pm 5,85$ & $25,83 \pm 3,76$ \\
\hline Valor de $p$ & & $p^{(1)}=0,875$ & $p^{(1)}=0,820$ \\
\hline \multirow[t]{3}{*}{ - $1000 \mathrm{~Hz}$} & Alfabetização & $11,00 \pm 6,52$ & $29,00 \pm 10,84$ \\
\hline & Primeira & $9,43 \pm 7,52$ & $30,71 \pm 8,86$ \\
\hline & Segunda/terceira & $9,17 \pm 4,92$ & $25,00 \pm 7,75$ \\
\hline Valor de $p$ & & $p^{(1)}=0,883$ & $p^{(1)}=0,533$ \\
\hline \multirow[t]{3}{*}{ - $2000 \mathrm{~Hz}$} & Alfabetização & $13,00 \pm 2,74^{(A)}$ & $27,00 \pm 8,37$ \\
\hline & Primeira & $5,14 \pm 4,60^{(B)}$ & $25,71 \pm 7,32$ \\
\hline & Segunda/terceira & $12,50 \pm 6,12^{(\mathrm{A})}$ & $28,33 \pm 6,06$ \\
\hline Valor de $p$ & & $p^{(1)}=0,017^{\star}$ & $p^{(1)}=0,811$ \\
\hline \multirow[t]{3}{*}{ - $4000 \mathrm{~Hz}$} & Alfabetização & $12,40 \pm 15,69$ & $21,00 \pm 9,62$ \\
\hline & Primeira & $10,57 \pm 7,91$ & $27,86 \pm 9,06$ \\
\hline & Segunda/terceira & $9,50 \pm 6,89$ & $27,50 \pm 6,89$ \\
\hline Valor de $p$ & & $p^{(1)}=0,898$ & $p^{(1)}=0,357$ \\
\hline
\end{tabular}

(1) - Através do teste Kruskal-Wallis. 
Tabela 5 - Estatísticas da média dos tempos de detecção de Gaps por freqüência, em função da ocorrência ou não de tratamento fonoaudiológico, no grupo com desvio fonológico

\begin{tabular}{lccc}
\hline \multirow{2}{*}{ Freqüência } & \multicolumn{2}{c}{ Tratamento com fono } & \multirow{2}{*}{ Valor de $\mathrm{p}$} \\
\cline { 2 - 3 } & Não & Sim & \\
\hline - $500 \mathrm{~Hz}$ & $25,00 \pm 4,08$ & $25,00 \pm 5,35$ & $\mathrm{p}^{(1)}=1,000$ \\
- $1000 \mathrm{~Hz}$ & $29,50 \pm 9,26$ & $26,88 \pm 8,84$ & $\mathrm{p}^{(1)}=0,551$ \\
- $2000 \mathrm{~Hz}$ & $27,50 \pm 7,55$ & $26,25 \pm 6,41$ & $\mathrm{p}^{(1)}=0,714$ \\
- $4000 \mathrm{~Hz}$ & $28,00 \pm 8,56$ & $23,13 \pm 8,43$ & $\mathrm{p}^{(1)}=0,244$ \\
\hline
\end{tabular}

$\left(^{\star}\right)$ - Diferença significante ao nível de 5,0\%.

(1) - Através do teste Mann-Whitney.

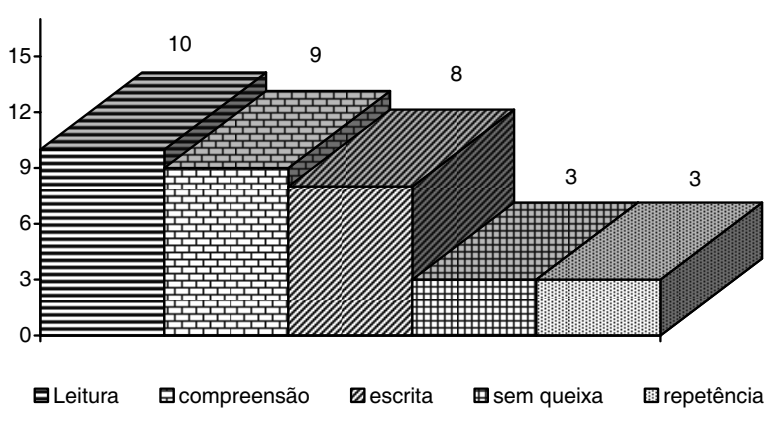

Figura 1 - Análise da ocorrência de dificuldade de aprendizado no grupo de portadores de desvio fonológico e processamento temporal alterado $(\mathrm{N}=17)$

tipo de dificuldade. Observa-se que a freqüência das dificuldades escolares apresentadas é grande, considerando-se a amostra pesquisada e as mais citadas foram: dificuldade de leitura (10), dificuldade de compreensão (9) e dificuldade na escrita (8).

\section{DISCUSSÃO}

Os resultados da análise do desempenho de processamento temporal no grupo com desvio fonológico, no grupo de crianças avaliadas, reforçam a corrente que afirma que sujeitos com distúrbios de linguagem necessitam de processamento temporal mais longo ${ }^{6,22}$ e apresentam dificuldades para discriminar, seqüencializar ou lembrar estímulos breves em uma sucessão rápida ${ }^{6,24,34}$. Tal fato se torna mais evidente ao se comparar com o desempenho deste grupo com o do grupo controle, que apresentou limiares de detecção dentro dos padrões normativos referidos pela literatura, inferiores a $20 \mathrm{~ms}^{17,18}$.

Considerando-se que para que a aquisição e o desenvolvimento normal da linguagem ocorram é necessário que haja integridade anátomo-fisiológica ${ }^{35}$, acredita-se que independente de sua origem, as alterações do processamento auditivo podem estar contidas em quadros de distúrbios de linguagem, piorando o desempenho de indivíduos em tarefas de compreensão do som que requeiram habilidades auditivas, assim como tais alterações podem atuar como causa das dificuldades de linguagem que ocorrem quando o indivíduo falha ao receber ou resgatar o que foi ouvido ${ }^{19,36}$.

A percepção auditiva contribui de forma muito importante para o desenvolvimento da fala e para o entendimento do que as pessoas falam; portanto, esta percepção alterada sempre leva à aquisição de problemas de comunicação ${ }^{28,29,37}$.

O processamento temporal está intimamente relacionado à percepção dos traços supra-segmentais da fala, uma vez que envolve as habilidades em perceber e armazenar estímulos acústicos não verbais. Considerando esta afirmativa, a percepção do estímulo temporal deficitária também pode levar a um baixo desempenho da habilidade de leitura e aprendizagem em geral ${ }^{22,34}$. Este fato pode ser comprovado nos dados obtidos para o grupo experimental (grupo 1) deste trabalho.

A prosódia e a entonação trazem emoção ao que está sendo falado (alegria, raiva, entusiasmo, surpresa, etc.) e pode mudar o significado da sentença. Uma vez que os indivíduos que apresentam processamento auditivo alterado não possuem uma boa discriminação de freqüência, intensidade e duração sonora, a prosódia e a entonação do que é falado provavelmente não são apreciadas por completo ${ }^{26}$.

A fala é um dos aspectos da compreensão no processo de comunicação. As falhas na percepção auditiva que interferem na fala ou atrapalham, de alguma maneira, a escolaridade podem estar associadas à imaturidade ou a problemas no desenvolvimento perceptivo. Por alguma razão, o processo de fala poderia estar comprometido em alguma habilidade como, por exemplo, na atenção (transmissão do sinal) ou na memória (análise, classificação, reconhecimento ou recomposição da seqüência) ${ }^{38}$. 
Embora haja consenso na literatura a respeito da relação dos transtornos do processamento auditivo e das alterações de linguagem, contrariando as expectativas, um trabalho realizado com 17 crianças com idades entre 6 e 10 anos, de ambos os sexos, portadoras de distúrbio de linguagem oral e escrita, as quais foram submetidas à avaliação do processamento temporal usando o Auditory Fusion Test-revised, mostrou que o tempo de respostas para o teste aplicado apresentou-se dentro do padrão normativo ${ }^{21}$.

É importante considerar a multiplicidade de fatores que podem estar relacionados às causas das alterações de linguagem e que estas, muitas vezes, podem não chegar a ser determinadas. Desta forma, embora muito freqüente, não é obrigatória a presença de transtornos do processamento auditivo associada às alterações de linguagem ${ }^{8}$, porém, a forte relação entre estes temas sustenta a teoria que aponta a necessidade de uma investigação mais abrangente na área da percepção auditiva, não se restringindo apenas aos aspectos periféricos.

Observando os dados obtidos neste estudo para o grupo controle no que toca à relação entre freqüência e tempo de detecção de Gaps observase que estes itens têm comportamento variável, corroborando a idéia do autor do teste ${ }^{17}$ que não encontrou diferenças significativas ao usar o RGDT nas freqüências de 500 a $4000 \mathrm{~Hz}$ e vão de encontro aos que acreditam que pela localização destas freqüências no sistema auditivo periférico, agudos na base e graves no ápice da cóclea, quanto mais aguda a freqüência maior o tempo necessário para a detecção de Gaps, uma vez que estará mais distante do sistema auditivo central ${ }^{18}$.

De maneira geral, na literatura pesquisada, não houve diferença significativa no estudo da variável sexo, concordando com os achados deste trabalho, o que leva a crer que este não é um ponto determinante para o desempenho do processamento auditivo temporal, quando avaliado com testes comportamentais ${ }^{18,39-41}$.

Considerando a idade dos indivíduos avaliados (6 a 9 anos), ao se analisar os dados obtidos, em ambos os grupos, e compará-los com os autores pesquisados, encontra-se concordância entre os achado obtidos e o que foi mencionado pela maioria deles $18,39,40$

No caso do processamento temporal, mais especificamente no que se refere ao tempo de detecção de Gaps aleatórios avaliado com o RGDT em crianças ouvintes normais com idade acima de 7 anos, parece não existir influência da idade, nem da série escolar. Tal fato leva a crer que as habilidades temporais aos sete anos já se apresenta bastante desenvolvida, sendo menos influenciada pelo aprendizado após os sete anos de idade ${ }^{18,39,40}$ e atinge seu ápice por volta dos doze anos ${ }^{23}$. Estas afirmativas são justificáveis pelo fato de que as habilidades auditivas temporais são processos importantes ocorridos nos seis a sete anos iniciais de vida ${ }^{42}$.

De uma forma geral, a maturação do sistema nervoso se dá no sentido caudal-rostral; desta forma, os tratos pré-talâmicos estão totalmente mielinizados por volta dos cinco a seis meses de vida. Entretanto, os tratos pós-talâmicos só atingem a completa mielinização por volta dos cinco a seis anos de idade. Já o corpo caloso e algumas áreas auditivas de associação podem não ter sua mielinização completa antes dos 10 a 12 anos de idade. A velocidade de mielinização nervosa varia; portanto, o desempenho de tarefas realizadas por estruturas que estão sendo mielinizadas também varia. Assim sendo, é necessária maturação para a realização de uma tarefa. Talvez seja esta a explicação para algumas diferenças de desempenho em tarefas de habilidades auditivas centrais ${ }^{43}$.

Provavelmente existe uma seqüência no desenvolvimento da percepção auditiva da fala e, as habilidades auditivas envolvidas, vão melhorando com o aumento da idade cronológica e o acúmulo de experiências acústicas ${ }^{44-46}$, porém, até certa faixa etária, estas diferenças podem ser mais evidentes. Uma criança normal, com idade de aproximadamente doze anos, apresenta o corpo caloso maduro, tendo respostas para os testes especiais do processamento auditivo próximas às dos adultos ${ }^{46-48}$.

Em estudo sobre a maturação do processamento auditivo, por meio de testes comportamentais em 60 crianças com dificuldades escolares, com idades entre 8 e 10 anos, foi constatada a influência da maturação auditiva no desempenho dos testes comportamentais (verbais), uma vez que as melhores respostas aparecem com o aumento da idade ${ }^{39}$.

Parece então que a natureza do estímulo pode ser um fator determinante de mudanças no desempenho do processamento auditivo e o que é observado para sons da fala (verbais) parece não ocorrer para sons não verbais ${ }^{18}$, o que justificaria a ausência de relação entre aumento de idade e melhora de desempenho do processamento temporal.

O fato é que as habilidades auditivas temporais são processos importantes ocorridos nos seis a sete anos iniciais de vida e contribuem para o conhecimento dos sons da fala, garantindo a aquisição e o aprendizado do sistema de linguagem. Problemas envolvendo o processamento auditivo podem afetar o aprendizado tanto em áreas de produção fonoarticulatória como de leitura e de escrita ${ }^{42}$. 
Diante de tal fato, existe a necessidade de identificação do problema assim que ele se inicia, propiciando um auxílio à criança para que adquira estratégias compensatórias, objetivando superar suas dificuldades, e o ideal é que o diagnóstico seja realizado o mais cedo possível para que não chegue a interferir no processo de escolaridade. Esta parece ser a opinião de muitos pesquisadores também ${ }^{9,42,43}$.

Os achados, em ambos os grupos, no que se refere à série escolar, revelam que esta não se apresenta como um fator importante para o desempenho do processamento temporal, uma vez que não se observam menores limiares de detecção de Gaps para as séries mais elevadas de maneira sistemática, concordando com estudos realizados na área ${ }^{18,40,41}$. Isto pode ocorrer pelo que já foi mencionado antes, ou seja, as habilidades temporais aos sete anos já se apresentam bem desenvolvidas, sendo menos influenciadas pela escolaridade.

Ao analisar a condição de ter feito tratamento fonoaudiológico ou não, no grupo com desvio fonológico, para nenhuma das freqüências comprovam-se diferenças significativas do ponto de vista estatístico, muito embora se tenha observado, de maneira geral, médias mais elevadas no grupo que realizou este tipo de tratamento. Estes achados podem ser creditados ao fato da terapia em questão não ter sido direcionada aos aspectos temporais da audição. Embora de maneira não significativa do ponto de vista estatístico, podem sugerir que a estimulação terapêutica pode ser uma alternativa válida para minimizar os transtornos de processamento auditivo, na esperança que também possam ser revertidas as suas conseqüências de maneira geral.

Observa-se que a freqüência das dificuldades escolares apresentadas é grande, considerando-se a amostra pesquisada. Estes dados apresentam-se de acordo com muitos estudos que citam a possibilidade de que problemas envolvendo o processamento auditivo possam afetar o aprendizado, tanto em áreas de produção fonoarticulatória como de leitura e de escrita $21,42,43,49$.

É comum encontrar entre as manifestações comportamentais indicativas da presença de um transtorno do processamento os distúrbios articulatórios; vocabulário inespecífico e ambíguo; sintaxe simplificada, erros e concordância; dificuldade na aprendizagem da leitura e escrita; distúrbios na aquisição de linguagem; dificuldade em manter a atenção a estímulos puramente auditivos, pedindo constantes repetições; tempo de latência aumentado para emissão de respostas e/ou emissão de respostas inconsistentes aos estímulos auditivos recebidos; dificuldade em compreender conceitos verbais e relacioná-los a conceitos visuais e/ou idéias abstratas; discriminação dos sons de fala prejudicada na presença ou não de estímulos simultâneos ou competitivos; falha na memorização das mensagens ouvidas; dificuldades na organização e seqüencialização de estímulos verbais e não-verbais e aprendizagem insuficiente quando restrita ao canal auditivo ${ }^{13,35,50}$.

Mesmo em sentido inverso, ou seja, ao pesquisar o processamento temporal em portadores de alterações de leitura e escrita, é possível encontrar uma forte relação com os transtornos de processamento auditivo ${ }^{51}$. A percepção auditiva tem sido relatada como uma das fontes de variação individual das habilidades fonológicas, que tem papel fundamental no aprendizado e no desenvolvimento da leitura; logo, a percepção do estímulo temporal deficitária pode levar a um baixo desempenho da habilidade de leitura e aprendizagem em geral ${ }^{22}$.

O processamento da fala envolve habilidades de processamento de alto nível e, de forma mais generalizada, as habilidades de processamento de linguagem e cognição ${ }^{52}$ e parece certo que prejuízos sutis no processamento auditivo das informações podem levar ao desenvolvimento de representações alteradas de informação verbal na memória e, ainda, a dificuldades de segmentação fonêmica de palavras, sendo que alteração da percepção auditiva não verbal pode afetar as habilidades fonológicas $^{24}$.

A aquisição da linguagem, oral ou escrita, está intimamente ligada às condições de escuta, muito embora a percepção auditiva não seja a sua fonte de informação. $O$ indivíduo está exposto desde muito cedo às nuances e variabilidades dos sons da língua materna e mesmo que ainda alcance o seu caráter formal, certamente já sofre as influências de seus aspectos supra-segmentais.

Pode-se dizer que os elementos prosódicos são vias de engajamento da criança no diálogo e, ao mesmo tempo, constituem fator coesivo na organização da forma fônica e nos princípios da estruturação fonológica ${ }^{52-54}$. Por esta razão, as alterações de percepção destes traços tão importantes para a aquisição dos aspectos formais da língua não podem ser desprezados.

Pensando que as crianças com desvio fonológico fazem parte de uma população heterogênea e com necessidades e limitações particulares a cada uma delas quanto às suas bagagens emocionais e ambientais, torna-se simplista tentar investigá-las por meio de um único instrumento. É, certamente, necessário um universo abrangente de informações para conhecer os fatores determinantes da sua dificuldade de aprendizagem da linguagem oral. 
O sentido da audição pode não ser o único a estar comprometido; no entanto, é recomendado não negligenciar a possibilidade do comprometimento do sistema auditivo central e/ou periférico nestes casos. Neste sentido, a investigação do processamento auditivo temporal pode contribuir com informações valiosas tanto para o diagnóstico quanto para o auxílio aos processos de aquisição uma vez que é adquirido muito cedo e se apresenta como pré-requisito para aquisição das estruturas formais da língua.

Considerando-se que as habilidades auditivas envolvem outros fatores como memória, atenção, motivação e outros, recomenda-se muita cautela durante a avaliação e a interpretação, sempre mantendo o foco nas questões singulares que possam vir a se apresentar. Por ser um procedimento de fácil aplicação e por trazer resultados significativos na diferenciação dos grupos estudados se propõe que a percepção dos sons não verbais, mais especificamente o processamento temporal, seja valorizada na avaliação e no acompanhamento de crianças com desvio fonológico.

\section{CONCLUSÃO}

Com base na avaliação das crianças participantes deste estudo e com objetivo de estudar o processamento temporal fazendo uso do teste de detecção de Gaps aleatórios (RGDT), foi possível observar que crianças com desvio fonológico podem apresentam alteração de processamento temporal e necessitam de mais tempo para detecção de intervalos de tempo entre estímulos auditivos do que as crianças sem desvio fonológico. De maneira geral, em ambos os grupos, não houve influência das variáveis: freqüência, sexo, idade, série escolar ou ocorrência de tratamento fonoaudiólogico. As queixas de aprendizado podem estar presentes nas crianças com desvio fonológico e alteração de processamento temporal.

\begin{abstract}
Purpose: to check the performance of temporal processes ability in children with phonological disorders. Methods: the study took place in a private university of Recife and the participants were 36 volunteers, with ages varying from 6 to 9 , of both genders and divided into two groups, with phonological disorders (experimental) and a control one. The data was collected using the RGDT (Keith) and the analysis made with the SPSS 13 by descriptive statistical techniques. Results: the results show higher levels of Gaps detection on the experimental; the difference between the groups was statistically significant for each frequency tested; there was no influence of the variables: periodicity, gender, age, school level; the speech therapy was not a determinant factor; the main referred learning difficulties were reading and comprehension problems. Conclusion: children with phonological disorders can present temporal processes disorders and need more time for intervals detection between auditory stimulations. In a generalized manner, in both groups, there was no difference as for the variable periodicity, gender, age, pertaining to school series or occurrence of speech treatment. The learning complaints can be found in children who have phonological and temporal processes disorders at the same time.
\end{abstract}

KEYWORDS: Hearing; Hearing Loss; Auditory Perception

\section{REFERÊNCIAS}

1. Allen MC, Schubert-Studia SE. Prevention of prelingual hearing impairment. Semin Hear. 1990; 11:134-48.

2. Musiek FE, Baran JA. Central auditory assessment: thirty years of challenge and change. Ear Hear. 1997; 8(4 Suppl.):22S-35S.

3. Phillips DP. Central auditory processing: a view from auditory neuroscience. Am J Otol. 1995; 16(3):338-52.

4. American Speech-Language-Hearing Association (ASHA) Task Force on Central Auditory Processing
Consensus Development. Central auditory processing: current status of research and implications for clinical practice. Am J Audiol. 1996; 5(2):41-54.

5. Osório MAM. Dificuldades de aprendizagem e perdas auditivas leves e moderadas. [monografia]. Goiânia (GO): CEFAC - Saúde e Educação; 1999.

6. Tallal P, Newcombe F. Impairment of auditory perception and language comprehension in dysphasia. Brain Lang. 1978; 5(1):13-34.

7. Junqueira CAO, Frizzo ACF. Potenciais evocados de curta, média e longa latência. In: Aquino AMCM. Processamento auditivo: eletrofisiologia e psicoacústica._São Paulo: Lovise; 2002. p. 63-86. 
8. Momensohn-Santos TM, Branco-Barreiro FCA. Avaliação e intervenção fonoaudiológica no transtorno de processamento auditivo. In: Ferreira LP, Befi-Lopes DM, Limongi SCO, organizador. Tratado de fonoaudiologia. São Paulo: Rocca; 2004. p. 554-67.

9. Pereira LD. Processamento auditivo central: abordagem passo a passo. In: Pereira LD, Schochat E. Processamento auditivo central: manual de avaliação. São Paulo: Lovise; 1997. p. 49-60.

10. Pinheiro ML, Musiek FE. Sequencing and temporal ordering in the auditory system. In: Keith RW. Assessment of central auditory dysfunction foundations and clinical correlates. Baltimore: Willians \& Wilkins; 1985. p. 219-38.

11. Basso SC, et al. Teste de padrões seqüenciais de duração em adultos do sexo feminino para diferentes tipos de respostas. In: IV Congresso Internacional de Fonoaudiologia e III Encontro Ibero-Americano de Fonoaudiologia, 1999, São Paulo. Anais, São Paulo; 1999. p. 254.

12. Kandel ER, Schwartz JH, Jessell TM. Fundamentos da neurociência e do comportamento. Rio de Janeiro: Guanabara Kookan; 2000.

13. Pereira LD, Cavadas M. Processamento auditivo central. In: Frota S. Fundamentos em fonoaudiologia-audiologia. Rio de Janeiro: Guanabara Koogan; 1998. p. 135-46.

14. Ferre JM. Processing power: a guide to CAPD assessment and management. San Antonio: Communication Skill Builders; 1997.

15. McPherson DL. Late potentials of the auditory system. San Diego: Singular Publishing Group; 1996.

16. Munhoz MSL, organizador. Audiologia clínica. São Paulo: Atheneu; 2000.

17. Keith RW. Manual of the random gap detection test. St. Louis: Auditec; 2000.

18. Barreto MASC, Muniz LF, Teixeira CF. Desempenho da habilidade de resolução temporal em crianças de 07 a 13 anos. Rev Soc Bras Fonoaudiol. 2004; 9(4):220-8.

19. Pereira LD. Identificação de desordem do processamento auditivo central através de observação comportamental: organização de procedimentos padronizados. In: Schochat L. Processamento auditivo. São Paulo: Lovise; 1996. p. 43-56.

20. Merzenich MM, Jenkins WM, Johnston P, Schreiner C, Miller SL, Tallal P. Temporal processing deficits of language-learning impaired children ameliorated by training. Science. 1996; 271(5245):77-81.

21. Feniman MR, Keith RW, Rebekah FC. Assessment of auditory processing in children with deficit hyperactivity disorder and language-based imparements. Rev Dist Commun. 1999; 11(1):9-27.
22. Pereira LD. Processamento auditivo: aspectos atuais. In: Encontro Internacional de Audiologia. São Paulo: Resumo de trabalhos apresentados; 2005. [cd-rom]

23. Balen SA. Processamento auditivo central: aspectos temporais da audição e percepção acústica da fala. [mestrado]. São Paulo (SP): Pontifícia Universidade Católica de São Paulo; 1996.

24. Mann VA, Brady S. Reading disability: the role of language deficiencies. J Consult Clin Psychol. 1988; 56(6):811-6.

25. Schochat E. Percepção de fala. In: Pereira LD, Schochat E, organizador. Processamento auditivo: série atualidades em fonoaudiologia. v. 2. São Paulo: Lovise; 1996. p. 15-42.

26. Thompson ME, Abel SM. Indices of hearing in patients with central auditory pathology II: choice response time. Scand Audiol Suppl. 1992; 35:17-22.

27. Azevedo MF. Programa de prevenção e identificação precoce dos distúrbios da audição. In: Schochat E, organizador. Processamento auditivo: série atualidades em fonoaudiologia. v. 2. São Paulo: Lovise; 1996. p. 75-105.

28. Wertzner HF. Fonologia: desenvolvimento e alterações. In: Ferreira LP, Befi-Lopes DM, Limongi SCO, organizador. Tratado de fonoaudiologia. São Paulo: Roca; 2004. p. 772-83.

29. Kent R. Auditory processing of speech. In: Katz J, Stecker NA, Henderson D. Central auditory processing: a transdisciplinary view. St. Louis: Mosby; 1992. p. 93-106.

30. Gonçalves CS. Variáveis lingüísticas facilitadoras na reabilitação fonológica das líquidas não-laterais. [mestrado]. Porto Alegre (RS): Pontifícia Universidade Católica do Rio Grande do Sul; 2002.

31. Schochat E, Rabelo CM, Sanfins MD. Processamento auditivo central: testes tonais de padrão de freqüência e de duração em indivíduos normais de 7 a 16 anos de idade. Pró-Fono; 2000; 12(2):1-7.

32. Musiek FE. Frequency (pitch) and duration patterns tests. J Am Acad Audiol. 1994; (5):265-8.

33. Felippe ACN. Processamento auditivo e problemas de leitura-escrita. In Aquino MCM, organizador. Processamento auditivo: eletrofisiologia e psicoacústica. São Paulo: Lovise; 2002. p. 102-8.

34. Jerger S, Jerger J. Alterações auditivas. São Paulo: Atheneu; 1989.

35. Alvarez AMM, Zaidan E. Disfunção não verbal. Acta AWHO. 2000; 19(1):49-55.

36. Azevedo MF. et al. Avaliação do processamento auditivo central: identificação de crianças de risco para alteração de linguagem e aprendizado durante o primeiro ano de vida. In: Marchesan IQ. et al. Tópicos em fonoaudiologia. São Paulo: Lovise; 1995. p. 447-62. 
37. Katz J. The SSW test: an interim report. J Speech Hear Dis. 1968; 3:132-337.

38. Machado SF. Avaliação da percepção da fala. São Paulo: Plexus; 1996.

39. Neves IF. Maturação do processamento auditivo em crianças com e sem dificuldades escolares. [mestrado]. São Paulo (SP): Faculdade de Medicina da Universidade de São Paulo; 2004.

40. Soares CD, Toniolo IMF. Habilidade de sequencialização sonora não verbal e verbal e de localização sonora em pré-escolares. Pró-Fono. 1998; 10(2):34-40.

41. Dornelles S. Orientação espacial, processamento auditivo e atenção visual em crianças com distúrbio da comunicação humana. [monografia]. São Paulo (SP): Escola Paulista de Medicina; 1994.

42. Santos MTM, Navas ALGP, Pereira LD. Estimulando a consciência fonológica. In: Pereira LD, Schochat E. Processamento auditivo central: manual de avaliação. São Paulo: Lovise; 1997. p. 85-90.

43. Chermark GD, Musiek FE. Central auditory processing disorders: new perspectives. London: Singular Publishing Group; 1997. p. 139-47.

44. Pereira LD, Ortiz KZ. Desordem do processamento auditivo central e distúrbios da produção fonoarticulatória. In: Lichting I, Carvallo RMM, organizador. Audição: abordagens atuais. São Paulo: Pró-fono; 1997. p. 173-86.

45. Koay MET. Speech and speech disorders: implications for central auditory processing. In: Katz J, Stecker NA, Henderson D. Central auditory processing: a transdiciplinary view. St. Louis: Mosby; 1992. p. 187-98.
46. Hall JW, Grose JH. Development of temporal resolution in children as measured by the temporal modulation transfer function. J Acoust Soc Am. 1994; 96(1):150-4.

47. Ballen SA. Processamento temporal: histórico, atualidades e perspectivas. In: Anais do Encontro Internacional de Audiologia, 20. São Paulo; 2005. p. 9.

48. Roeser RJ, Downs MP. Auditory disorders in school children. 2. ed. New York: Thieme Medical Publisher; 1988.

49. Watson BU. Auditory temporal processing and reading disability. J Acoust Soc Am. 1988; 84(1): S76.

50. Katz J. Classification of auditory processing disorders. In: Central auditory processing: a transdiciplinary view. St. Louis: Mosby Year Books; 1992.

51. Watson BU, Miller TK. Auditory perception, phonological processing, and reading ability/disability. J Speech Hear Res. 1993; 36(4):850-63.

52. Duchan JF, Katz J. Language and auditory processing: top-down plus bottom-up. In: Lasky EZ, Katz J. Central auditory processing disorders: problems of speech and language learning. Austin: Pro-Ed; 1983. p. 31-48.

53. Scarpa EM. Entonação e processos dialógicos: fusão ou diferenciação? In: Lemos C, organizador. Aquisição da linguagem. Série Estudos. Uberaba: Faculdades Integradas de Uberaba; 1985. p. 56-74.

54. Scarpa EM. The development of intonation and dialogue processes. In: Snow C, Conti-Ramsden G, organizador. Children's language, vol. 7. Hildale: Lawrence Erlbaum; 1990.

Endereço para correspondência:

Rua Dom José Lopes, 665, ap. 1401

Recife - PE

CEP: $51020-3170$

Tel: (81) 33251281 / 91383399

Fax: (81) 34161636

E-mail: lilianmuniz@ @erra.com.br 